\title{
On the coarse-grained evolution of collisionless stellar systems
}

\author{
P. H. Chavanis ${ }^{1}$ and F. Bouchet ${ }^{2}$ \\ ${ }^{1}$ Laboratoire de Physique Théorique, Université Paul Sabatier, 118 route de Narbonne, 31062 Toulouse, France \\ e-mail: chavanis@irsamc.ups-tlse.fr \\ 2 École Normale Supérieure de Lyon, 46 allée d'Italie, 69364 Lyon, France \\ e-mail: Freddy. Bouchet@ens-lyon.fr
}

Received 14 June 2004 / Accepted 8 September 2004

\begin{abstract}
We describe the dynamical evolution of collisionless stellar systems on a coarse-grained scale. We first discuss the statistical theory of violent relaxation, following the seminal paper of Lynden-Bell (1967). Consistently with this statistical approach, we present kinetic equations for the coarse-grained distribution function $\bar{f}(\boldsymbol{r}, \boldsymbol{v}, t)$ based on a Maximum Entropy Production Principle or on a quasi-linear theory of the Vlasov-Poisson system. Then, we develop a deterministic approach where the coarse-grained distribution function is defined as a convolution of the fine-grained distribution function $f(\boldsymbol{r}, \boldsymbol{v}, t)$ by a Gaussian window. We derive the dynamical equation satisfied by $\bar{f}(\boldsymbol{r}, \boldsymbol{v}, t)$ and show that its stationary states are different from those predicted by the statistical theory of violent relaxation. This implies that the notion of coarse-graining must be defined with care. We apply these results to the HMF (Hamiltonian Mean Field) model and find that the spatial density is similar to a Tsallis $q$-distribution where the $q$ parameter is related to the resolution length.
\end{abstract}

Key words. Galaxy: kinematics and dynamics - gravitation - stellar dynamics

\section{Introduction}

For most stellar systems, including the important class of elliptical galaxies, the relaxation time due to close encounters is larger than the Hubble time by several orders of magnitude (Binney \& Tremaine 1987). Therefore, close encounters are negligible and the fundamental dynamics is that of a collisionless system in which the constituent particles (stars) move under the influence of the mean potential generated by all the other particles. Mathematically, the dynamics of stellar systems is described by the self-consistent Vlasov-Poisson system. The evolution of the Vlasov-Poisson system is extremely complicated. Although the dynamics is collisionless, the fluctuations of the gravitational potential are able to redistribute energy between stars and provide an effective relaxation mechanism on a very short timescale (violent relaxation). This collisionless relaxation is able to account for the regularity of elliptical galaxies and other astrophysical bodies.

Starting from an unstable initial condition, the VlasovPoisson system develops an intricate filamentation in phase space (phase mixing). In physical space, this mixing process is associated with the heavily damped oscillations of a galaxy away from mechanical equilibrium. In a strict sense, the distribution function $f(\boldsymbol{r}, \boldsymbol{v}, t)$ does not reach an equilibrium distribution but develops smaller and smaller filaments. However, if we introduce a coarse-graining procedure and locally average over the filaments, the coarse-grained distribution function $\bar{f}(\boldsymbol{r}, \boldsymbol{v}, t)$ is expected to reach a stationary state $\bar{f}(\boldsymbol{r}, \boldsymbol{v})$ on a short timescale of the order of the dynamical time. It is usually advocated that this metaequilibrium state is a particular stationary solution of the Vlasov equation.

A statistical theory appropriate for this process of violent relaxation has been developed by Lynden-Bell (1967). For given initial condition, this theory predicts the most mixed state consistent with the constraints imposed by the dynamics (mass, energy and Casimirs integrals). Unfortunately, the statistical prediction of Lynden-Bell is limited by the problem of incomplete relaxation. Real stellar systems tend towards the equilibrium state during violent relaxation but cannot attain it: the fluctuations of the gravitational potential die away before the relaxation process is complete (Lynden-Bell 1967). For that reason, Tremaine et al. (1987) introduce the notion of $H$-functions in order to quantify the importance of mixing. They show in particular that these functionals increase on the coarse-grained scale while the coarse-grained energy is approximately conserved. Therefore, the metaequilibrium state maximizes a certain $H$-function at fixed mass and energy. This is a particular stationary solution of the Vlasov equation with nonlinear dynamical stability (Chavanis 2003a,b; Chavanis \& Sire 2004). 
In this paper, we discuss the dynamical evolution of collisionless stellar systems. Although the evolution of the finegrained distribution function $f(\boldsymbol{r}, \boldsymbol{v}, t)$ is entirely determined by the Vlasov-Poisson system, we are in general more interested by the evolution of the coarse-grained distribution function $\bar{f}(\boldsymbol{r}, \boldsymbol{v}, t)$. The problem thus consists of determining the dynamical equation satisfied by $\bar{f}$. In a previous paper, Chavanis et al. (1996) have obtained such an equation by using a phenomenological Maximum Entropy Production Principle (MEPP). This principle assumes that, during violent relaxation, the system tends to maximize the rate of entropy production $\dot{S}$ while conserving all the constraints imposed by the dynamics. It leads to a generalized Fokker-Planck equation involving a diffusion in velocity space and friction. The friction is nonlinear so as to account for the specific constraints associated with collisionless evolution. On the other hand, the diffusion coefficient, which is related to the fluctuations of the distribution function, can account for the process of incomplete relaxation. As the fluctuations weaken when the system approaches equilibrium, the coarse-grained relaxation is stopped and the system can remain frozen in a confined region of phase space. This dynamical effect can physically solve the infinite mass problem associated with the maximization of the Boltzmann entropy (in the sense of Lynden-Bell) in an unlimited domain. Another approach consists of developing a quasilinear theory of the Vlasov-Poisson system (Kadomtsev \& Pogutse 1970; Severne \& Luwel 1980; Chavanis 2002a, 2004). This approach is expected to describe a "gentle relaxation" valid close to the equilibrium state when the fluctuations have weakened. It leads to a generalized Landau equation respecting the specificities of the collisionless relaxation. In the two-level approximation of the theory of violent relaxation, this kinetic equation relaxes towards the Fermi-Dirac distribution function predicted by Lynden-Bell (1967). The generalization to an arbitrary spectrum of phase levels is also possible.

In the quasilinear theory, the smooth distribution function $\bar{f}$ results from two operations of averaging: a local average over a macrocell of size $\epsilon_{r}^{3} \epsilon_{v}^{3}$ and a statistical average over different realizations of the flow. We shall refer to this situation as the statistical approach. It is closely related to the statistical theory of Lynden-Bell. In this paper, we shall consider a different approach. We define the coarse-grained distribution as the convolution of $f(\boldsymbol{r}, \boldsymbol{v}, t)$ by a Gaussian window of size $\epsilon_{r}^{3} \epsilon_{v}^{3}$. We come back therefore to the original definition of "coarsegraining". As we shall see, this approach leads to a new type of kinetic equation which differs from the one obtained with the quasilinear theory or with the MEPP. We shall refer to this situation as the deterministic approach because it just exploits the properties of the Gaussian window and it does not make any assumption about stochastic fluctuations.

Our new treatment has been inspired by the work of Bouchet (2004) in the context of two-dimensional turbulence. As already observed in previous works, the 2D Euler-Poisson system presents deep analogies to the Vlasov-Poisson system (see Chavanis 2002b). Therefore, it is of interest to put the two topics in parallel and investigate how the results obtained in one domain can be extended to the other.

\section{The statistical approach}

\subsection{The maximum entropy production principle}

Basically, a collisionless stellar system is described by the Vlasov-Poisson system

$\frac{\partial f}{\partial t}+\boldsymbol{v} \frac{\partial f}{\partial \boldsymbol{r}}+\boldsymbol{F} \frac{\partial f}{\partial \boldsymbol{v}}=0$

$\Delta \Phi=4 \pi G \int f \mathrm{~d}^{3} \boldsymbol{v}$,

where $\boldsymbol{F}=-\boldsymbol{\nabla} \Phi$ is the gravitational force by unit of mass (acceleration). We introduce the decomposition $f=\bar{f}+\tilde{f}$ where $\bar{f}$ is the coarse-grained distribution function and $\tilde{f}$ is the fluctuation. We assume that $\bar{f}$ results from a statistical average so that $\overline{\bar{f}}=\bar{f}$. Taking the local average of the Vlasov equation, we obtain an equation of the form

$\frac{\partial \bar{f}}{\partial t}+v \frac{\partial \bar{f}}{\partial \boldsymbol{r}}+\overline{\boldsymbol{F}} \frac{\partial \bar{f}}{\partial \boldsymbol{v}}=-\frac{\partial \boldsymbol{J}}{\partial \boldsymbol{v}}$

for the coarse-grained distribution function, with a diffusion current $\boldsymbol{J}=\overline{\tilde{f} \tilde{\boldsymbol{F}}}$ related to the correlations of the fine-grained fluctuations. There is no diffusion in position space since the velocity $v$ is a pure coordinate and therefore does not fluctuate.

The problem in hand consists of determining the current $\boldsymbol{J}$. One suggestion is to use a phenomenological Maximum Entropy Production Principle (Chavanis et al. 1996). In the simplest case where the fine-grained distribution function takes only two values $f=\eta_{0}$ and $f=0$, it leads to an equation of the form

$\frac{\partial \bar{f}}{\partial t}+L \bar{f}=\frac{\partial}{\partial \boldsymbol{v}}\left\{D\left[\frac{\partial \bar{f}}{\partial \boldsymbol{v}}+\beta(t) \bar{f}\left(\eta_{0}-\bar{f}\right) \boldsymbol{v}\right]\right\}$,

where $L=\boldsymbol{v} \partial / \partial \boldsymbol{r}+\overline{\boldsymbol{F}} \partial / \partial \boldsymbol{v}$ is the advection operator in phase space and $D$ the diffusion coefficient. The inverse temperature

$\beta(t)=-\frac{\int D \frac{\partial \bar{f}}{\partial \boldsymbol{v}} \boldsymbol{v} \mathrm{d}^{3} \boldsymbol{r} \mathrm{d}^{3} \boldsymbol{v}}{\int D \bar{f}\left(\eta_{0}-\bar{f}\right) v^{2} \mathrm{~d}^{3} \boldsymbol{r} \mathrm{d}^{3} \boldsymbol{v}}$

evolves in time so as to satisfy the conservation of energy $\dot{E}=0$. Morphologically, this equation can be viewed as a generalized Fokker-Planck equation (or Kramers equation) involving a diffusion in velocity space and friction (Chavanis $2003 \mathrm{~b}$ ). We note that the friction is nonlinear due to the product $\bar{f} \times\left(\eta_{0}-\bar{f}\right)$ which guarantees that $\bar{f} \leq \eta_{0}$, as imposed by the Vlasov equation on the coarse-grained distribution function. Therefore, the relaxation Eq. (4) converges towards the Lynden-Bell distribution

$\bar{f}=\frac{\eta_{0}}{1+\lambda \mathrm{e}^{\beta \eta_{0}\left(\frac{v^{2}}{2}+\Phi\right)}}$,

which coincides with the Fermi-Dirac distribution in the twolevel approximation (Chavanis \& Sommeria 1998).

The relaxation Eq. (4) possesses several interesting properties. However, the MEPP approach is phenomenological and does not provide the value of the diffusion coefficient $D$. 
Secondly, it leads to an equation which is not Galilean invariant. In particular, the friction depends on the velocity of a stellar fluid particle, not on the relative velocity, as we could expect. Finally, the conservation of energy is enforced by a formal Lagrange multiplier $\beta(t)$ whose physical interpretation is not clear. Therefore, the status of the MEPP is not firmly established. Yet, it can provide a useful parametrization of mixing in the nonlinear regime of the violent relaxation where perturbative methods such as the quasilinear theory of Sect. 2.2 fail. In any case, the generalized relaxation equations obtained with the MEPP can serve as numerical algorithms to compute the statistical equilibrium state predicted by Lynden-Bell (1967) (this can have interesting applications for other systems with long-range interactions described by the Vlasov equation). To improve the model, the relaxation equations can be extended so as to satisfy the conservation, at the fine-grained scale, of all the Casimirs (Chavanis et al. 1996). Moreover, the diffusion coefficient can be related to the fluctuations of the distribution function. In that case, it vanishes when the fluctuations die away (Chavanis 1998). This effect should lead to a confinement of the system in phase space and avoid the infinite mass problem.

It may also be of interest to introduce a relaxation equation which monotonically increases a $H$-function $S=$ $-\int C(f) \mathrm{d}^{3} \boldsymbol{r} \mathrm{d}^{3} \boldsymbol{v}$, where $C$ is convex, while conserving mass and energy. This is associated with the phenomenology of violent relaxation where the $H$-functions are weak constraints (i.e. affected by the coarse-graining) while the energy is a robust constraint (i.e. approximately conserved at the coarsegrained scale). Such a relaxation has the form of a generalized Fokker-Planck equation (Chavanis 2003b):

$\frac{\partial \bar{f}}{\partial t}+L \bar{f}=\frac{\partial}{\partial \boldsymbol{v}}\left\{D\left[\frac{\partial \bar{f}}{\partial \boldsymbol{v}}+\frac{\beta(t)}{C^{\prime \prime}(\bar{f})} v\right]\right\}$

It satisfies $\dot{S} \geq 0$ and leads to a stationary state of the form

$C^{\prime}(f)=-\beta \epsilon-\alpha$,

which maximizes the $H$-function $S$ at fixed $M$ and $E$. Therefore, the relaxation Eq. (7) can be used as a numerical algorithm to construct any nonlinearly dynamically stable stationary solution of the Vlasov equation of the form $f=F(\beta \epsilon+\alpha)$ with $F(x)=\left(C^{\prime}\right)^{-1}(-x)$ specified by the function $C$. We can thus obtain a larger class of stationary solutions of the Vlasov equation that are not captured by the statistical mechanics of violent relaxation due to incomplete relaxation. Similar equations can be written in 2D turbulence (Robert \& Sommeria 1992; Chavanis 2003b) and for other systems with long-range interactions described by the Vlasov equation.

\subsection{The quasilinear theory}

Another kinetic theory of violent relaxation was developed by Kadomtsev \& Pogutse (1970), Severne \& Luwel (1980) and Chavanis $(2002 a, 2004)$ by using a quasilinear theory of the Vlasov-Poisson system. Subtracting Eqs. (1) and (3), we obtain the following equation for the fluctuations

$$
\frac{\partial \tilde{f}}{\partial t}+\boldsymbol{v} \frac{\partial \tilde{f}}{\partial \boldsymbol{r}}+\overline{\boldsymbol{F}} \frac{\partial \tilde{f}}{\partial \boldsymbol{v}}=-\tilde{\boldsymbol{F}} \frac{\partial \bar{f}}{\partial \boldsymbol{v}}-\tilde{\boldsymbol{F}} \frac{\partial \tilde{f}}{\partial \boldsymbol{v}}+\overline{\tilde{\boldsymbol{F}} \frac{\partial \tilde{f}}{\partial \boldsymbol{v}}} .
$$

The essence of the quasilinear theory is to assume that the fluctuations are weak and neglect the nonlinear terms in Eq. (9) altogether. Thus, this theory can only describe the late stages of the process of violent relaxation when the fluctuations have weakened.

When this approximation is used, it is possible to solve Eq. (9) formally in terms of Green functions to obtain the fluctuating distribution function $\tilde{f}$ in term of the past evolution of $\bar{f}$. Substituting the resulting expression for $\tilde{f}$ in Eq. (3) and implementing a Markovian approximation, a local approximation and a linear trajectory approximation, the current can be explicitly evaluated. In the two-level approximation, this approach leads to a kinetic equation of the form

$$
\begin{aligned}
& \frac{\partial \bar{f}}{\partial t}+L \bar{f}= \\
& \frac{\partial}{\partial v^{\mu}} \int \mathrm{d}^{3} \boldsymbol{v}^{\prime} K^{\mu v}\left[\bar{f}^{\prime}\left(\eta_{0}-\bar{f}^{\prime}\right) \frac{\partial \bar{f}}{\partial v^{\nu}}-\bar{f}\left(\eta_{0}-\bar{f}\right) \frac{\partial \bar{f}^{\prime}}{\partial v^{\prime \nu}}\right]
\end{aligned}
$$

with

$K^{\mu v}=2 \pi G^{2} \epsilon_{r}^{3} \epsilon_{v}^{3} \ln \left(\frac{R}{\epsilon_{r}}\right) \frac{u^{2} \delta^{\mu v}-u^{\mu} u^{v}}{u^{3}}$,

where $\bar{f}^{\prime}$ stands for $\bar{f}\left(\boldsymbol{r}, \boldsymbol{v}^{\prime}, t\right)$ and $\boldsymbol{u}=\boldsymbol{v}^{\prime}-\boldsymbol{v}$ is the relative velocity. Equation (10) can be viewed as a generalized Landau equation. As before, this equation involves a term $\bar{f} \times\left(\eta_{0}-\bar{f}\right)$, which enforces the constraint $\bar{f} \leq \eta_{0}$, and converges at equilibrium towards the Lynden-Bell distribution function (6). Contrary to the MEPP approach, this equation is self-consistent, Galilean invariant, and the conservation of energy results from properties of symmetry and not from a formal Lagrange multiplier. A connexion can be found between Eqs. (4) and (10) if we replace $f^{\prime}$ in Eq. (10) by its equilibrium value (6). This may be a reasonable approximation if we are close to equilibrium (Chavanis 1998). Nevertheless, the degree of validity of this approximation is difficult to estimate. A quasilinear theory of the 2D Euler equation leading to a kinetic equation akin to Eq. (10) has also been developed in the context of 2D turbulence (Chavanis 2000).

We can also propose a generalization of Eq. (10) which increases an arbitrary $H$-function (Chavanis 2004):

$$
\frac{\partial \bar{f}}{\partial t}+L \bar{f}=\frac{\partial}{\partial v^{\mu}} \int \mathrm{d}^{3} \boldsymbol{v}^{\prime} K^{\mu v}\left[\frac{1}{C^{\prime \prime}\left(\bar{f}^{\prime}\right)} \frac{\partial \bar{f}}{\partial v^{v}}-\frac{1}{C^{\prime \prime}(\bar{f})} \frac{\partial \bar{f}^{\prime}}{\partial v^{\prime v}}\right] .
$$

This can be viewed as a heuristic approach to go beyond the two-level approximation while leaving the problem tractable. A more rigorous approach would be to write down a set of $N$ coupled relaxation equations for each level $\eta$ as sketched by Severne \& Luwel (1980). 


\section{The deterministic approach}

\subsection{The coarse-grained Vlasov equation}

We shall now develop a completely different approach to the process of coarse-grained relaxation. We define the local average $\bar{h}(\boldsymbol{r}, \boldsymbol{v})$ of a quantity $h(\boldsymbol{r}, \boldsymbol{v})$ by the convolution

$\bar{h}(\boldsymbol{r}, \boldsymbol{v})=\int h(\boldsymbol{r}+\boldsymbol{s}, \boldsymbol{v}+\boldsymbol{w}) G(\boldsymbol{s}, \boldsymbol{w}) \mathrm{d}^{3} \boldsymbol{s} \mathrm{d}^{3} \boldsymbol{w}$

where

$G(s, w)=\frac{1}{\left(2 \pi \epsilon_{r} \epsilon_{v}\right)^{3}} \mathrm{e}^{-s^{2} / 2 \epsilon_{r}^{2}} \mathrm{e}^{-w^{2} / 2 \epsilon_{v}^{2}}$

is a Gaussian window in phase space. If $h(\boldsymbol{r}, \boldsymbol{v})$ has a filamentary structure at small scales, this operation smoothes out this function on a phase space cell of size $\epsilon_{r}^{3} \epsilon_{v}^{3}$. We also define the fluctuation as $\tilde{h}=h-\bar{h}$. Contrary to the statistical average considered in Sect. 2, the averaging operator (14) does not verify the relation $\overline{\bar{f}}=\bar{f}$. Therefore, new terms arise when we average the Vlasov equation with Eq. (14).

We can write the locally averaged Vlasov equation in the form

$\frac{\partial \bar{f}}{\partial t}+L \bar{f}=-\frac{\partial \boldsymbol{J}_{\mathrm{d} r}}{\partial \boldsymbol{r}}-\frac{\partial}{\partial \boldsymbol{v}}\left(\boldsymbol{J}_{\mathrm{d} v}+\boldsymbol{J}_{\mathrm{s}}\right)$.

The current has been split in two parts. The terms $\boldsymbol{J}_{\mathrm{d} r}=\bar{f} \boldsymbol{v}-\bar{f} \boldsymbol{v}$ and $\boldsymbol{J}_{\mathrm{d} v}=\overline{f \overline{\boldsymbol{F}}}-\bar{f} \overline{\boldsymbol{F}}$ which do not depend on the fluctuations of the gravitational field form the deterministic component of the current. These terms reduce to zero for a statistical average. In the present context, they represent the dominant contribution to the current. The term $\boldsymbol{J}_{\mathrm{s}}=\overline{f \tilde{\boldsymbol{F}}}$ which depends on the fluctuations of the gravitational field forms the stochastic component of the current. It is similar to the term considered in Sect. 2.

We shall now express the deterministic component of the current in terms of the coarse-grained distribution function in order to obtain a closed kinetic equation. First of all, we note that

$\boldsymbol{J}_{\mathrm{d} r}=\int f(\boldsymbol{r}+\boldsymbol{s}, \boldsymbol{v}+\boldsymbol{w}, t) G(\boldsymbol{s}, \boldsymbol{w}) \boldsymbol{w} \mathrm{d}^{3} \boldsymbol{s} \mathrm{d}^{3} \boldsymbol{w}$.

Using the property of the Gaussian window, this can be rewritten

$\boldsymbol{J}_{\mathrm{d} r}=-\epsilon_{v}^{2} \int f(\boldsymbol{r}+\boldsymbol{s}, \boldsymbol{v}+\boldsymbol{w}, t) \frac{\partial G}{\partial \boldsymbol{w}} \mathrm{d}^{3} \boldsymbol{s} \mathrm{d}^{3} \boldsymbol{w}$.

Integrating by parts, we finally obtain

$\boldsymbol{J}_{\mathrm{d} r}=\epsilon_{v}^{2} \frac{\partial \bar{f}}{\partial \boldsymbol{v}}$

To simplify $\boldsymbol{J}_{\mathrm{d} v}$, we expand the smooth gravitational force $\overline{\boldsymbol{F}}$ in Taylor series around $\boldsymbol{r}$. To leading order, we get

$J_{\mathrm{d} v}^{i}=\frac{\partial \bar{F}_{i}}{\partial x_{j}} \int f(\boldsymbol{r}+\boldsymbol{s}, \boldsymbol{v}+\boldsymbol{w}, t) G(\boldsymbol{s}, \boldsymbol{w}) s_{j} \mathrm{~d}^{3} \boldsymbol{s} \mathrm{d}^{3} \boldsymbol{w}$.

Now, using a procedure similar to that leading to Eq. (18) we obtain

$J_{\mathrm{d} v}^{i}=-\epsilon_{r}^{2} \frac{\partial^{2} \bar{\Phi}}{\partial x_{i} \partial x_{j}} \frac{\partial \bar{f}}{\partial x_{j}}$.
Collecting the previous results, the equation for the coarsegrained distribution function can be put in the form

$$
\frac{\partial \bar{f}}{\partial t}+L \bar{f}=\left(\epsilon_{r}^{2} \frac{\partial^{2} \bar{\Phi}}{\partial x_{i} \partial x_{j}}-\epsilon_{v}^{2} \delta_{i j}\right) \frac{\partial^{2} \bar{f}}{\partial x_{i} \partial v_{j}} .
$$

To obtain this equation, we have used an approximation and a method similar to that developed by Bouchet (2004) in the case of the 2D Euler equation. In this analogy, the term in parenthesis in Eq. (21) plays the same role as the strain tensor in $2 \mathrm{D}$ hydrodynamics.

\subsection{The conservation laws}

We shall now discuss the conservation laws associated with the coarse-grained Vlasov equation. The coarse-grained energy is

$E=\frac{1}{2} \int \overline{f v^{2}} \mathrm{~d}^{3} \boldsymbol{r} \mathrm{d}^{3} \boldsymbol{v}+\frac{1}{2} \int \overline{\rho \Phi} \mathrm{d}^{3} \boldsymbol{r}=K+W$,

where $K$ is the kinetic energy and $W$ the potential energy. Expanding the expression

$\overline{f v^{2}}=\int f(\boldsymbol{r}+\boldsymbol{s}, \boldsymbol{v}+\boldsymbol{w}, t)(\boldsymbol{v}+\boldsymbol{w})^{2} G(\boldsymbol{s}, \boldsymbol{w}) \mathrm{d}^{3} \boldsymbol{s} \mathrm{d}^{3} \boldsymbol{w}$,

we find that

$\overline{f v^{2}}=\bar{f} v^{2}+I_{1}+I_{2}$

where

$I_{1}=2 \boldsymbol{v} \cdot \int f(\boldsymbol{r}+\boldsymbol{s}, \boldsymbol{v}+\boldsymbol{w}, t) w G(\boldsymbol{s}, \boldsymbol{w}) \mathrm{d}^{3} \boldsymbol{s} \mathrm{d}^{3} \boldsymbol{w}$,

$I_{2}=\int f(\boldsymbol{r}+\boldsymbol{s}, \boldsymbol{v}+\boldsymbol{w}, t) w^{2} G(\boldsymbol{s}, \boldsymbol{w}) \mathrm{d}^{3} \boldsymbol{s} \mathrm{d}^{3} \boldsymbol{w}$.

The first integral is equal to

$I_{1}=2 \epsilon_{v}^{2} v \cdot \frac{\partial \bar{f}}{\partial v}$

To compute the second integral, we use the fact that

$w^{2} G=\epsilon_{v}^{4} \Delta_{v} G+3 \epsilon_{v}^{2} G$.

Substituting this identity in Eq. (26) and integrating by parts twice, we finally obtain

$I_{2}=\epsilon_{v}^{4} \Delta_{v} \bar{f}+3 \epsilon_{v}^{2} \bar{f}$.

Regrouping these results, we find that the coarse-grained kinetic energy is

$K=\int \bar{f} \frac{v^{2}}{2} \mathrm{~d}^{3} v-\frac{3}{2} \epsilon_{v}^{2} M$,

where $M=\int \bar{f} \mathrm{~d}^{3} \boldsymbol{r} \mathrm{d}^{3} \boldsymbol{v}$ is the mass. For the potential energy, we have

$W=\frac{1}{2} \int \overline{\rho \bar{\Phi}} \mathrm{d}^{3} \boldsymbol{r}+\frac{1}{2} \int \overline{\rho \tilde{\Phi}} \mathrm{d}^{3} \boldsymbol{r}$.

The second term represents the energy of the stochastic fluctuations $\boldsymbol{J}_{\mathrm{s}}$ (see Eq. (15)). Consistent with the neglect of $\boldsymbol{J}_{\mathrm{s}}$, 
we will neglect this contribution to the energy. Since the coarsegrained potential $\bar{\Phi}$ is smooth, it can be expanded in a Taylor series. Using a method similar to that of Sect. 3.1, we obtain to leading order in $\epsilon_{r}$,

$W=\frac{1}{2} \epsilon_{r}^{2} \int \nabla \bar{\rho} \cdot \nabla \bar{\Phi} \mathrm{d}^{3} \boldsymbol{r}+\frac{1}{2} \int \bar{\rho} \bar{\Phi} \mathrm{d}^{3} \boldsymbol{r}$

Now, integrating by parts and using the Poisson equation, the first integral can be rewritten

$\int \nabla \bar{\rho} \cdot \nabla \bar{\Phi} \mathrm{d}^{3} \boldsymbol{r}=-4 \pi G M_{2}$

where

$M_{2}=\int \bar{\rho}^{2} \mathrm{~d}^{3} \boldsymbol{r}$.

In conclusion, the coarse-grained energy is

$E=\int \bar{f} \frac{v^{2}}{2} \mathrm{~d}^{3} \boldsymbol{v} \mathrm{d}^{3} \boldsymbol{r}+\frac{1}{2} \int \bar{\rho} \bar{\Phi} \mathrm{d}^{3} \boldsymbol{r}-\frac{3}{2} \epsilon_{v}^{2} M-2 \pi G \epsilon_{r}^{2} M_{2}$.

The coarse-grained angular momentum is

$\boldsymbol{L}=\int \overline{f \boldsymbol{r} \times \boldsymbol{v}} \mathrm{d}^{3} \boldsymbol{r} \mathrm{d}^{3} \boldsymbol{v}$.

Using an adaptation of the preceding results, we find that

$\overline{f x_{i} v_{j}}=\bar{f} x_{i} v_{j}+\epsilon_{r}^{2} \frac{\partial \bar{f}}{\partial x_{i}} v_{j}+\epsilon_{v}^{2} x_{i} \frac{\partial \bar{f}}{\partial v_{j}}+\epsilon_{r}^{2} \epsilon_{v}^{2} \frac{\partial^{2} \bar{f}}{\partial x_{i} \partial v_{j}}$.

Since the derivatives vanish upon integration, we finally find that the coarse-grained angular momentum is

$\boldsymbol{L}=\int \bar{f} \boldsymbol{r} \times \boldsymbol{v} \mathrm{d}^{3} \boldsymbol{r} \mathrm{d}^{3} \boldsymbol{v}$

The coarse-grained impulse is

$\boldsymbol{P}=\int \overline{f \boldsymbol{v}} \mathrm{d}^{3} \boldsymbol{r} \mathrm{d}^{3} \boldsymbol{v}$.

Since

$\overline{f v}=\bar{f} v+\epsilon_{v}^{2} \frac{\partial \bar{f}}{\partial v}$,

we finally find that the coarse-grained impulse is

$\boldsymbol{P}=\int \bar{f} \boldsymbol{v} \mathrm{d}^{3} \boldsymbol{r} \mathrm{d}^{3} \boldsymbol{v}$.

Because the energy, the angular momentum and the impulse are conserved by the Vlasov equation, the coarse-grained energy, angular momentum and impulse must be conserved by the coarse-grained Vlasov Eq. (21). We directly verify this property in Appendix A.

\subsection{The H-functions}

Following Tremaine et al. (1987), we introduce a family of functionals, called $H$-functions, defined by

$S=-\int C(f) \mathrm{d}^{3} \boldsymbol{r} \mathrm{d}^{3} \boldsymbol{v}$,

where $C$ is convex. These functionals are particular Casimirs, so they are conserved by the Vlasov equation. However, Tremaine et al. (1987) show that they increase on the coarsegrained scale in the sense that $S\left[\bar{f}\left(t_{2}\right)\right] \geq S\left[\bar{f}\left(t_{1}\right)\right]$, where it is assumed that, at $t=t_{1}$, the system is not mixed, i.e. $\bar{f}\left(\boldsymbol{r}, \boldsymbol{v}, t_{1}\right)=f\left(\boldsymbol{r}, \boldsymbol{v}, t_{1}\right)$. Due to the resemblance with the $H$-theorem in kinetic theory, $S$ is sometimes called a generalized entropy (Chavanis 2003b). However, we emphasize that the $H$-functions do not have a statistical origin (they cannot be obtained from a counting analysis), that they are not universal (contrary to the Boltzmann entropy in statistical mechanics), and that a monotonic increase of $S(t)$ is not granted in general.

Using our approach, we can determine the evolution of $S(t)$ via the coarse-grained Vlasov Eq. (21). After straightforward integration by parts, we obtain

$\dot{S}=-\int C^{\prime \prime}(\bar{f}) \frac{\partial \bar{f}}{\partial x_{i}}\left(\epsilon_{r}^{2} \frac{\partial^{2} \bar{\Phi}}{\partial x_{i} \partial x_{j}}-\epsilon_{v}^{2} \delta_{i j}\right) \frac{\partial \bar{f}}{\partial v_{j}} \mathrm{~d}^{3} \boldsymbol{r} \mathrm{d}^{3} \boldsymbol{v}$.

We note that the coarse-graining operation (13) is compatible with the symmetry $t \rightarrow-t, \boldsymbol{v} \rightarrow-\boldsymbol{v}$. As a consequence, the coarse-grained Vlasov Eq. (21) remains reversible. Therefore, no Lyapunov functional exists and the $H$-functions cannot monotonically increase for every initial conditions. This is at variance with the kinetic equations introduced in the statistical approach of Sect. 2 which admit a Lyapunov functional.

\subsection{The stationary distribution}

The stationary solutions of Eq. (21) satisfy the differential equation

$\boldsymbol{v} \frac{\partial \bar{f}}{\partial \boldsymbol{r}}+\overline{\boldsymbol{F}} \frac{\partial \bar{f}}{\partial \boldsymbol{v}}=\left(\epsilon_{r}^{2} \frac{\partial^{2} \bar{\Phi}}{\partial x_{i} \partial x_{j}}-\epsilon_{v}^{2} \delta_{i j}\right) \frac{\partial^{2} \bar{f}}{\partial x_{i} \partial v_{j}}$.

This is an integro-differential equation since $\bar{\Phi}$ depends on $\bar{f}$ through the Poisson Eq. (2). We look for a particular stationary solution of the coarse-grained Vlasov equation in the form

$\bar{f}=\mathrm{e}^{-\beta\left(\frac{\nu^{2}}{2}+\bar{\Phi}(\boldsymbol{r})\right)} h(\boldsymbol{r})$.

If the function $h$ satisfies the equation

$\frac{\partial h}{\partial x_{j}}=-\beta\left(\epsilon_{r}^{2} \frac{\partial^{2} \bar{\Phi}}{\partial x_{i} \partial x_{j}}-\epsilon_{v}^{2} \delta_{i j}\right)\left[\frac{\partial h}{\partial x_{i}}-\beta h \frac{\partial \bar{\Phi}}{\partial x_{i}}\right]$,

then Eq. (45) is a solution of Eq. (44). We shall solve this equation perturbatively for $\epsilon_{r}, \epsilon_{v} \rightarrow 0$. We thus consider the expansion

$h=A+\epsilon_{r}^{2} h_{r}+\epsilon_{v}^{2} h_{v}+\ldots$

$\bar{\Phi}=\bar{\Phi}_{0}+\epsilon_{r}^{2} \bar{\Phi}_{r}+\epsilon_{v}^{2} \bar{\Phi}_{v}+\ldots$ 
To zeroth order, we have

$\bar{f}=A \mathrm{e}^{-\beta\left(\frac{v^{2}}{2}+\bar{\Phi}_{0}(\boldsymbol{r})\right)}$.

To the next order, the functions $h_{r}$ and $h_{v}$ are determined by the differential equations

$\frac{\partial h_{r}}{\partial x_{j}}=A \beta^{2} \frac{\partial^{2} \bar{\Phi}_{0}}{\partial x_{i} \partial x_{j}} \frac{\partial \bar{\Phi}_{0}}{\partial x_{i}}$,

$\frac{\partial h_{v}}{\partial x_{j}}=-A \beta^{2} \frac{\partial \bar{\Phi}_{0}}{\partial x_{j}}$

These equations are easily integrated and we obtain

$\bar{f}(\boldsymbol{r}, \boldsymbol{v})=A \mathrm{e}^{-\beta\left(\frac{\nu^{2}}{2}+\bar{\Phi}(\boldsymbol{r})\right)}\left\{1+\frac{1}{2} \epsilon_{r}^{2} \beta^{2}(\nabla \bar{\Phi})^{2}-\epsilon_{v}^{2} \beta^{2} \bar{\Phi}\right\}$.

This result is valid up to order $\epsilon^{2}$. The function $\bar{\Phi}$ (which has to be computed to first order in the exponential) is determined by the Poisson Eq. (2) with $f$ given by Eq. (52).

We note that the distribution function (52) is not a function of the energy $\epsilon=\frac{v^{2}}{2}+\bar{\Phi}$ alone. This is at variance with the statistical prediction of Lynden-Bell (1967) that leads to

$\bar{f}(\boldsymbol{r}, \boldsymbol{v})=\frac{\int \chi(\eta) \mathrm{e}^{-(\beta \epsilon+\alpha) \eta} \eta \mathrm{d} \eta}{\int \chi(\eta) \mathrm{e}^{-(\beta \epsilon+\alpha) \eta} \mathrm{d} \eta}=\bar{f}(\epsilon)$,

where $\chi(\eta)$ is a function determined by the initial conditions (see, e.g., Chavanis 2003a). This shows that the definition of the coarse-grained distribution function is of crucial importance as it determines the form of the stationary state. We emphasize that Eq. (52) is not neither a stationary solution of the Vlasov equation since the smooth advective part $L \bar{f}=$ $\boldsymbol{v} \partial \bar{f} / \partial \boldsymbol{r}+\overline{\boldsymbol{F}} \partial \bar{f} / \partial \boldsymbol{v}$ is counter-balanced by an effective "collision" term arising from coarse-graining. This differs from the MEPP and from the quasilinear theory described in Sect. 2 where both the advective term and the collision term cancel out individually at equilibrium.

Another difference between the two approaches is that Eq. (21) is reversible while Eqs. (4) and (10) are not. Therefore, Eqs. (4) and (10) are robust against small perturbations and converge towards a unique stationary distribution since a Lyapunov functional (entropy) exists. In contrast, the coarsegrained Vlasov Eq. (21) is smooth but keeps the deterministic aspect of the Vlasov equation. As such, it can converge towards a large class of stationary solutions (recall that Eq. (52) is just a particular solution of Eq. (21)) since there is no associated Lyapunov functional.

\section{The HMF model}

The results presented previously are relatively general. They apply to other systems described by the Vlasov equation, for different types of potential of interaction. Thus, it can be of interest to test these ideas on simpler systems. The Hamiltonian Mean Field (HMF) model has recently received growing attention in statistical mechanics as it exhibits features similar to gravitational systems while being more easily tractable analytically and numerically (see, e.g., Dauxois et al. 2002; Chavanis et al. 2004).
The HMF model describes the motion of particles on a circle interacting via a cosine binary potential $u\left(x-x^{\prime}\right)=$ $\frac{k}{2 \pi} \cos \left(x-x^{\prime}\right)$ where $k$ is the coupling constant ( $x$ denotes here the angular position of a particle on the circle). This is probably the simplest model with long-range interactions. It is onedimensional and corresponds to the truncation to one mode of the Fourier expansion of a potential $u\left(x-x^{\prime}\right)$. Mathematically, the HMF model is defined by the Hamilton equations

$\frac{\mathrm{d} x_{i}}{\mathrm{~d} t}=\frac{\partial H}{\partial v_{i}}, \quad \frac{\mathrm{d} v_{i}}{\mathrm{~d} t}=-\frac{\partial H}{\partial x_{i}}$,

where $H$ is the Hamiltonian

$H=\sum_{i=1}^{N} \frac{1}{2} v_{i}^{2}-\frac{k}{4 \pi} \sum_{i \neq j} \cos \left(x_{i}-x_{j}\right)$.

For fixed $t$ and $N \rightarrow+\infty$, it can be shown that the evolution of the system is described by the Vlasov equation

$\frac{\partial f}{\partial t}+v \frac{\partial f}{\partial x}+F \frac{\partial f}{\partial v}=0$

where $F=-\Phi^{\prime}$ with

$\Phi(x, t)=-\frac{k}{2 \pi} \int_{0}^{2 \pi} \cos \left(x-x^{\prime}\right) \rho\left(x^{\prime}, t\right) \mathrm{d} x^{\prime}$.

In one dimension, the coarse-grained Vlasov Eq. (21) becomes

$\frac{\partial \bar{f}}{\partial t}+v \frac{\partial \bar{f}}{\partial x}+\bar{F} \frac{\partial \bar{f}}{\partial v}=\left(\epsilon_{r}^{2} \frac{\partial^{2} \bar{\Phi}}{\partial x^{2}}-\epsilon_{v}^{2}\right) \frac{\partial^{2} \bar{f}}{\partial x \partial v}$.

Looking for stationary solutions in the form $f=g(v) h(x)$, we find that

$g(v)=\mathrm{e}^{-\beta \frac{v^{2}}{2}}$,

while $h(x)$ satisfies a differential equation of the form

$\frac{\mathrm{d} h}{\mathrm{~d} x}+\frac{\bar{\Phi}^{\prime}}{\frac{1}{\beta}-\epsilon_{v}^{2}+\epsilon_{r}^{2} \bar{\Phi}^{\prime \prime}} h=0$.

This equation is easily integrated and we finally obtain

$\bar{f}=A \mathrm{e}^{-\beta \frac{v^{2}}{2}} \exp \left\{-\beta \int \frac{\bar{\Phi}^{\prime} \mathrm{d} x}{1-\beta\left(\epsilon_{v}^{2}-\epsilon_{r}^{2} \bar{\Phi}^{\prime \prime}\right)}\right\}$.

This result is valid for an arbitrary potential of the form $u(x-$ $\left.x^{\prime}\right)$. More explicit results can be obtained for the HMF model with cosine interactions. From Eq. (57), we have $\Phi^{\prime \prime}=-\Phi$. Thus, the distribution function (61) becomes

$\bar{f}=A \mathrm{e}^{-\beta \frac{v^{2}}{2}} \exp \left\{-\beta \int \frac{\bar{\Phi}^{\prime} \mathrm{d} x}{1-\beta\left(\epsilon_{v}^{2}+\epsilon_{r}^{2} \bar{\Phi}\right)}\right\}$.

The integral can be performed explicitly and we get

$\bar{f}=A \mathrm{e}^{-\beta \frac{v^{2}}{2}}\left[1-\beta\left(\epsilon_{v}^{2}+\epsilon_{r}^{2} \bar{\Phi}\right)\right]^{1 / \epsilon_{r}^{2}}$.

Considering the limit $\epsilon \rightarrow 0$, one recovers the perturbative result (52). On the other hand, integrating on the velocity, we find that the density is related to the potential by

$\bar{\rho}=A^{\prime}\left[1-\beta\left(\epsilon_{v}^{2}+\epsilon_{r}^{2} \bar{\Phi}\right)\right]^{1 / \epsilon_{r}^{2}}$. 
This can be viewed as a $q$-distribution of the form

$\rho=\left[\lambda-\frac{q-1}{T q} \Phi\right]^{\frac{1}{q-1}}$

where $q=1+\epsilon_{r}^{2} \geq 1$ is related to the resolution length. These distributions have been recently investigated in connexion with Tsallis (1988) generalized thermodynamics. In the present context, this is just a coincidence because there is no direct relation to thermodynamics. This shows that the $q$-distributions arise in different domains of physics for reasons that do not necessarily have a link to statistical mechanics and thermodynamics. We already reached this conclusion in other occasions (Chavanis 2003b, 2004; Chavanis \& Sire 2004). Restricting ourselves to symmetric solutions, we have $\Phi=B \cos x$ so that

$\rho(x)=A^{\prime}\left[1-\beta\left(\epsilon_{v}^{2}+\epsilon_{r}^{2} B \cos x\right)\right]^{1 / \epsilon_{r}^{2}}$.

\section{Conclusion}

In this paper, we have discussed the coarse-grained evolution of collisionless stellar systems described by the Vlasov equation. The main conclusion of our work is that the concepts of coarse-graining and violent relaxation are complicated and subtle. We have presented two types of approaches which lead to very different results. In the statistical approach, we obtain kinetic equations similar to generalized Fokker-Planck and Landau equations. These equations monotonically increase a mixing entropy and relax towards the statistical equilibrium state predicted by Lynden-Bell's theory of violent relaxation. The effect of incomplete relaxation appears in the diffusion coefficient, related to the fluctuations of the distribution function, that can freeze the evolution of the system towards the maximum entropy state. This can solve the infinite mass problem. In the deterministic approach, the coarse-grained distribution function is defined as a local average (in phase space) of the fine-grained distribution function by a Gaussian window. This leads to a new type of kinetic equation, Eq. (21). This equation respects the conservation of energy, angular momentum and impulse on the coarse-grained scale but does not admit any Lyapunov functional. Furthermore, the stationary states of this equation differ from the statistical prediction of Lynden-Bell. They are not neither stationary solutions of the Vlasov equation because the advective term is precisely balanced by the "effective" collision term arising from coarsegraining. This approach is purely determinisitic and should describe short time-scale complex evolution of a given initial condition. Presumably, a more relevant model should also take into account the stochastic character of the evolution of an assembly of initial conditions. The ideas presented in this paper can have applications for other systems with long-range interactions such as two-dimensional vortices and the HMF model. The dynamical stability of collisionless stellar systems described by the coarse-grained Vlasov equation will be studied in a forthcoming paper (in preparation).

\section{Appendix A: Conservation of the coarse-grained constraints}

Let us verify explicitly that the coarse-grained energy (35), angular momentum (38) and impulse (41) are conserved by the coarse-grained Vlasov Eq. (21). We have

$\dot{E}=\int \frac{\partial \bar{f}}{\partial t} \frac{v^{2}}{2} \mathrm{~d}^{3} \boldsymbol{v} \mathrm{d}^{3} \boldsymbol{r}+\int \frac{\partial \bar{f}}{\partial t} \bar{\Phi} \mathrm{d}^{3} \boldsymbol{v} \mathrm{d}^{3} \boldsymbol{r}-2 \pi G \epsilon_{r}^{2} \dot{M}_{2}$

Using Eq. (21) and integrating by parts, we get

$\dot{E}=\epsilon_{r}^{2} \int \frac{\partial \Delta \bar{\Phi}}{\partial x_{j}} v_{j} \bar{f} \mathrm{~d}^{3} \boldsymbol{r} \mathrm{d}^{3} \boldsymbol{v}-2 \pi G \epsilon_{r}^{2} \dot{M}_{2}$.

Using the Poisson equation and integrating by parts one more time, we obtain

$\dot{E}=-4 \pi G \epsilon_{r}^{2} \int \bar{\rho} \nabla(\bar{\rho} \boldsymbol{u}) \mathrm{d}^{3} \boldsymbol{r}-2 \pi G \epsilon_{r}^{2} \dot{M}_{2}$,

where the local velocity $\boldsymbol{u}(\boldsymbol{r}, t)$ is defined by

$\bar{\rho} \boldsymbol{u}(\boldsymbol{r}, t)=\int \bar{f} \boldsymbol{v} \mathrm{d}^{3} \boldsymbol{v}$.

Integrating the coarse-grained Vlasov Eq. (21) over velocity leads to the continuity equation

$\frac{\partial \bar{\rho}}{\partial t}+\nabla(\bar{\rho} \boldsymbol{u})=0$.

Inserting this result in Eq. (A.3), we obtain

$\dot{E}=4 \pi G \epsilon_{r}^{2} \int \bar{\rho} \frac{\partial \bar{\rho}}{\partial t} \mathrm{~d}^{3} \boldsymbol{r}-2 \pi G \epsilon_{r}^{2} \dot{M}_{2}=0$.

For the coarse-grained angular momentum, we have

$\dot{L}_{k}=\int \epsilon_{i j k} \frac{\partial \bar{f}}{\partial t} x_{i} v_{j} \mathrm{~d}^{3} \boldsymbol{r} \mathrm{d}^{3} \boldsymbol{v}$.

Using Eq. (21) and integrating by parts, we get

$\dot{L}_{k}=-\int \epsilon_{i j k} \chi_{m j} \frac{\partial \bar{f}}{\partial x_{m}} x_{i} \mathrm{~d}^{3} \boldsymbol{r} \mathrm{d}^{3} \boldsymbol{v}$,

where we have defined

$\chi_{i j}=\epsilon_{r}^{2} \frac{\partial^{2} \bar{\Phi}}{\partial x_{i} \partial x_{j}}-\epsilon_{v}^{2} \delta_{i j}$.

Integrating by parts one more time, we obtain

$\dot{L}_{k}=\int \epsilon_{i j k} \bar{f}\left(\chi_{i j}+\epsilon_{r}^{2} x_{i} \frac{\partial \Delta \bar{\Phi}}{\partial x_{j}}\right) \mathrm{d}^{3} \boldsymbol{r} \mathrm{d}^{3} \boldsymbol{v}$.

Using the Poisson equation, the foregoing expression is equivalent to

$\dot{L}_{k}=\int \epsilon_{i j k} \bar{\rho} \chi_{i j} \mathrm{~d}^{3} \boldsymbol{r}+2 \pi G \epsilon_{r}^{2} \int \epsilon_{i j k} x_{i} \frac{\partial \bar{\rho}^{2}}{\partial x_{j}} \mathrm{~d}^{3} \boldsymbol{r}$.

Integrating the second term by parts and using $\epsilon_{i i k}=0$, we are left with

$\dot{L}_{k}=\epsilon_{r}^{2} \int \epsilon_{i j k} \bar{\rho} \frac{\partial^{2} \bar{\Phi}}{\partial x_{i} \partial x_{j}} \mathrm{~d}^{3} \boldsymbol{r}$. 
Therefore,

$\dot{\boldsymbol{L}}=\epsilon_{r}^{2} \int \bar{\rho} \nabla \times(\nabla \bar{\Phi}) \mathrm{d}^{3} \boldsymbol{r}=\mathbf{0}$.

Finally, for the coarse-grained impulse, we have

$\dot{P}_{k}=\int \frac{\partial \bar{f}}{\partial t} v_{k} \mathrm{~d}^{3} \boldsymbol{r} \mathrm{d}^{3} \boldsymbol{v}$.

Using Eq. (21) and integrating by parts, we get

$\dot{P}_{k}=-\int \chi_{i k} \frac{\partial \bar{f}}{\partial x_{i}} \mathrm{~d}^{3} \boldsymbol{r} \mathrm{d}^{3} \boldsymbol{v}=\epsilon_{r}^{2} \int \bar{\rho} \frac{\partial \Delta \bar{\Phi}}{\partial x_{k}} \mathrm{~d}^{3} \boldsymbol{r}$.

Using the Poisson equation, we obtain

$\dot{\boldsymbol{P}}=2 \pi G \epsilon_{r}^{2} \int \nabla\left(\bar{\rho}^{2}\right) \mathrm{d}^{3} \boldsymbol{r}=\mathbf{0}$.

\section{References}

Binney, J., \& Tremaine, S. 1987, Galactic Dynamics, Princeton Series in Astrophysics

Bouchet, F. 2004, Phys. Fluids, submitted [cond-mat/0305205]

Chavanis, P. H. 1998, MNRAS, 300, 981

Chavanis, P. H. 2000, PRL, 84, 5512
Chavanis, P. H. 2002a, Statistical mechanics of violent relaxation in stellar systems, Proc. Conf. Multiscale Problems in Science and Technology (Springer 2002) [arXiv: astro-ph/0212205]

Chavanis, P. H. 2002b, Statistical mechanics of two-dimensional vortices and stellar systems, Dynamics and thermodynamics of systems with long range interactions, ed. T. Dauxois, S. Ruffo, E. Arimondo, \& M. Wilkens, Lecture Notes in Physics (Springer) [cond-mat/0212223]

Chavanis, P. H. 2003a, A\&A, 401, 15

Chavanis, P. H. 2003b, PRE, 68, 6108

Chavanis, P. H. 2004, Physica A, 332, 89

Chavanis, P. H., \& Sommeria, J. 1998, MNRAS, 296, 569

Chavanis, P. H., Vatteville, J., \& Bouchet, F. 2004, [cond-mat/0408117]

Chavanis, P. H., Sommeria, J., \& Robert, R. 1996, ApJ, 471, 385

Chavanis, P. H., \& Sire, C. 2004, [cond-mat/0409569]

Dauxois, T., Latora, V., Rapisarda, A., Ruffo, S., \& Torcini, A. 2002, The Hamiltonian Mean Field Model: from Dynamics to Statistical Mechanics and back, Dynamics and thermodynamics of systems with long range interactions, ed. T. Dauxois, S. Ruffo, E. Arimondo, \& M. Wilkens, Lecture Notes in Physics (Springer) [cond-mat/0208456]

Kadomtsev, B. B., \& Pogutse, O. P. 1970, PRL, 25, 1155

Lynden-Bell, D. 1967, MNRAS, 136, 101

Robert, R., \& Sommeria, J. 1992, PRL, 69, 2776

Severne, G., \& Luwel, M. 1980, Ap\&SS, 72, 293

Tremaine, S., Hénon, M., \& Lynden-Bell, D. 1987, MNRAS, 227, 543

Tsallis, C. 1988, J. Stat. Phys., 52, 479 